\title{
Study of water droplet evaporation in a mini- channel with gas flow
}

\author{
Evgeniy Orlik ${ }^{1,2^{*}}$ and Ekaterina Isachenko ${ }^{1,3}$ \\ ${ }^{1}$ Institute of Thermophysics SB RAS, 630090, Novosibirsk, Russia \\ ${ }^{2}$ Novosibirsk State Agricultural University, 630039, Novosibirsk, Russia \\ ${ }^{3}$ Novosibirsk State University, 630090, Novosibirsk, Russia
}

\begin{abstract}
A series of experiments was conducted to study the evaporation of single water droplets with volume of $80 \sim 150 \mu 1$ on the polished stainless steel substrate in temperature range of the substrate surface of $25-80^{\circ} \mathrm{C}$ in 6 $\mathrm{mm}$ high mini-channel with gas flow and the Reynolds number of the gas flow within $0-2500$. New data on the water drop evaporation depending on the temperature of gas were obtained. The shadow method was the main method of measurement. As a result of experiments the experimental dependences for evaporation were obtained, and the influence of the gas flow rate and temperature on the water drop evaporation was analyzed.
\end{abstract}

\section{Introduction}

Further development of the microelectronic and power technology, which is widely used in the creation of modern ecological transport, in aircraft and space industry, is hampered by a serious technical problem of removal of high local heat fluxes, emitted on the chip and electronic elements. By far the most effective method of heat removal is based on the use of the phase transition and is directly related to the liquid evaporation. Spray cooling by the flow of droplets shows high efficiency of heat removal [1], but the very process of liquid drop evaporation is still not fully understood. Droplet evaporation has been in detail studied both numerically and experimentally, but mostly in open atmosphere. Contact angle of wetting and its hysteresis are the dominant parameters of evaporation [2-4]. Experimental studies show that the maximum heat removal is realized from the contact line [5], whose thickness is about tens microns [6]. In [7] it is shown that in the evaporation of volatile liquids, it is necessary to consider thermocapillary effects leading to the deformation of the interface and contact line. The influence of gas flow on the droplet evaporation is known, however all related studies were conducted in the open air with insufficient controlled conditions [8]. Present paper is devoted to the detailed investigation of the evaporation of water droplet placed on a heated substrate in a flat mini-channel with air flow. The experimental results obtained may be used to develop a new method of heat remvoal on the basis of near-wall drop motion.

*Corresponding author: evgeniy@gmail.com 


\section{Experimental setup and methods}

\subsection{Experimental setup}

The scheme of experimental setup is shown in figure 1. It includes a channel with height varying from $4 \mathrm{~mm}$ to $20 \mathrm{~mm}$, removable substrates, control of the substrate temperature, and the air supply system. The side walls and the top cover are equipped with optical windows for visualization. The aim of this work is to study the influence of substrate temperature and Reynolds number of the air flow on the drop evaporation rate for a channel with $6 \mathrm{~mm}$ height. The working fluid is ultrapure water, obtained with a Milli-Q system. The drop was placed into the channel with a syringe. The research was carried out at the initial drop volume of $60-150 \mathrm{~mm}$. All experiments were performed on a substrate of stainless steel with polished surface. The Reynolds number of the gas flow varied from 0 to 2000. The temperature in the air flow was maintained equal by means of a system of thermoregulation. Heating of the substrate was carried out by Peltier elements installed in contact with the substrate. The substrate temperature during the experiment was regulated with a precision of up to 0.20 using the controller the PR-59.

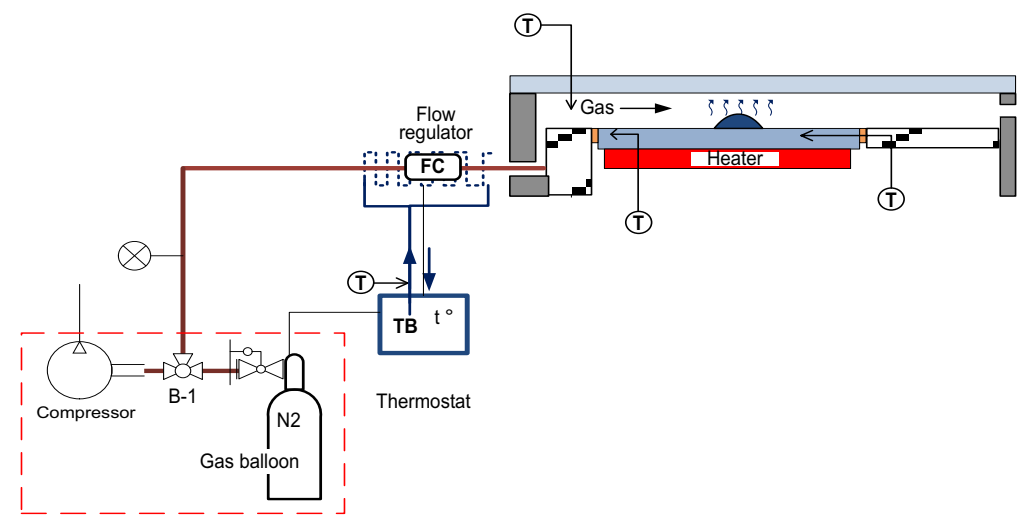

Fig. 1. Scheme of the experimental setup.

\subsection{Methods}

To register the contour of a sitting drop on a solid substrate we used the shadow method. Its principle is based on the fact that the physical object is illuminated by a parallel light beam, and its shadow is recorded by the camera, as shown in figure 2. Optical equipment allows obtaining images with a resolution of $6 \mu \mathrm{m} /$ pixel (see figure 4). The obtained images were processed by different methods with the help of software (the Drop Shape Analysis by KRÜSS). This software has multiple methods for stydying the drop contour. This work used the method of height/width to calculate the height of the drop and the diameter of its base, and the elliptic method for calculating the droplet volume during the experiment.

The first stage was the estimation of the error of calculating the drop volume with the elliptic method (see Fig. 2b) and 3). With this view using the rotary device DSA-100 the angle of the substrate inclination to the horizon was changed. As a result, under the action of gravity the drop became asymmetric, thereby the process of the droplet blow-off the in the gas flow was simulated as shown in figure $2 \mathrm{~b}$ ). From the obtained results it may be concluded that the difference between the advancing and receding angles of the droplet is less than $10^{\circ}$, and the error of volume measurement is $5 \%$. 

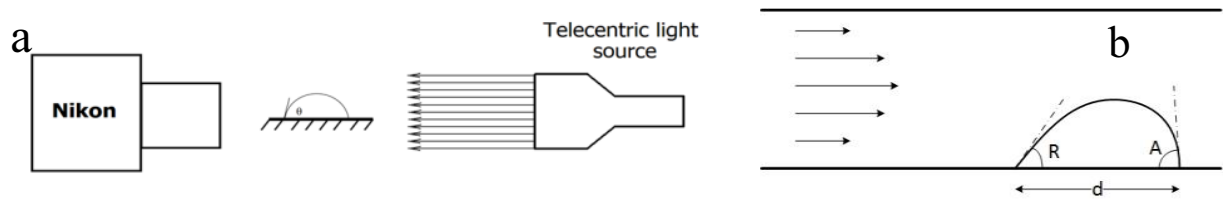

Fig. 2. a) The shadow method, b) The scheme of the drop.

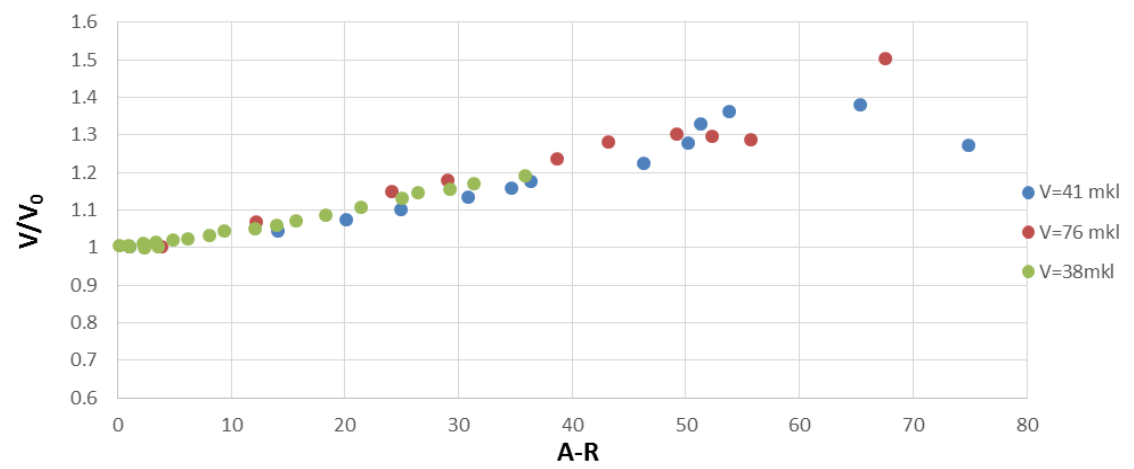

Fig. 3. Estimating the error of the drop volume calculation by the elliptic method in the software "The drop shape analysis", $\mathrm{V}$ is the current volume of the drop, $\mathrm{V}_{0}$ is the drop volume at the substrate inclination by $0^{\circ}, \mathrm{A}$ - advancing angle, and $\mathrm{R}$ - receding angle.

\section{Results}

Figure 4 shows photos of the process of drop evaporation at temperatures of the substrate 50 and $70^{\circ}$ and two different flow rates. Registration of images was conducted with 10 second interval between frames.

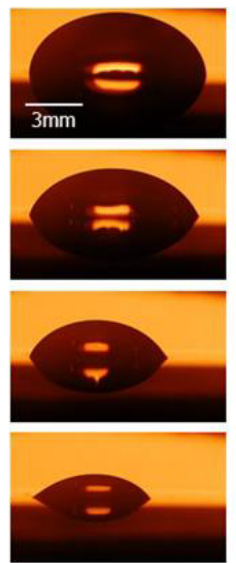

$\mathrm{T}=50 \mathrm{C}$ Flow $=10 \mathrm{I} / \mathrm{min}$
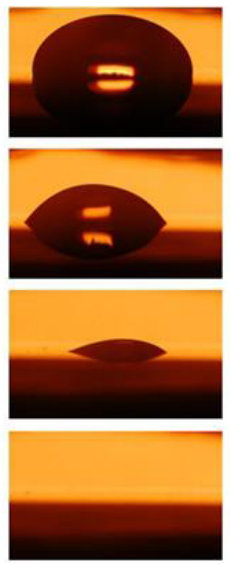

$\mathrm{T}=50 \mathrm{C}$

Flow $=70 \mathrm{I} / \mathrm{min}$
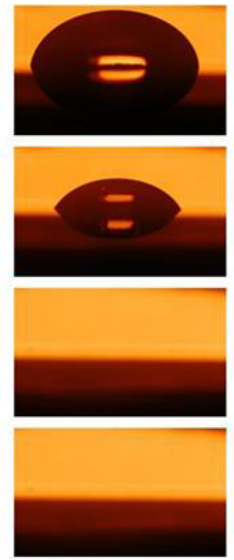

$\mathrm{T}=70 \mathrm{C}$

Flow $=10 \mathrm{l} / \mathrm{min}$
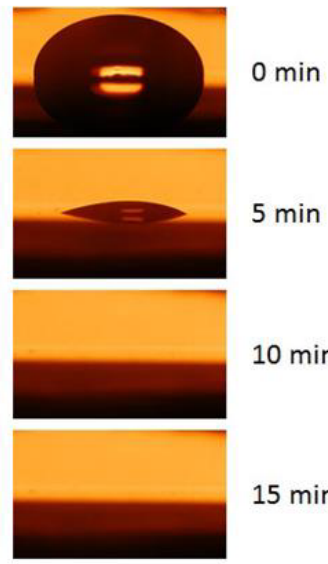

$10 \mathrm{~min}$

$\mathrm{T}=70 \mathrm{C}$

Flow $=70 \mathrm{l} / \mathrm{min}$

Fig. 4. Droplet evaporation depending on the substrate temperature and gas flow rate.

Evolution of droplet volume has been done in figure 5. It seems that life time of droplet can be used for generalization of evolution of droplet volume. Air temperature has a little 
effect for evaporation as obvious because low thermal conductivity of air, life time is almost the same for green and blue symbols.

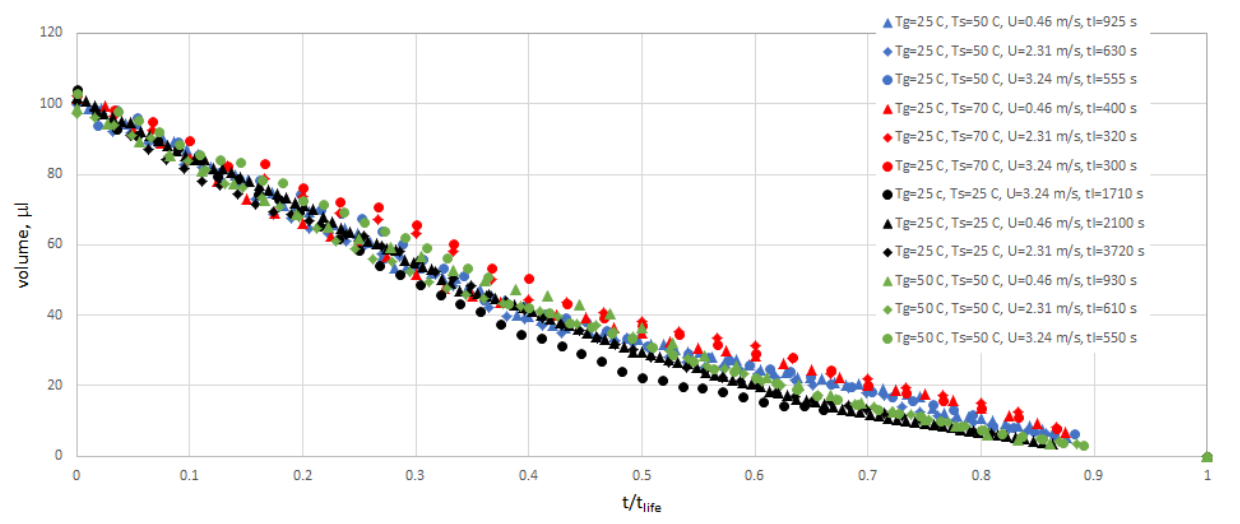

Fig. 5. Droplet volume versus $t / t_{\text {life }}$, subscripts: 1 - life time, $s$ - substrate, $g-$ gas. $V_{\text {init }} \sim 100 \mu 1$.

Figure 6 shows data for the evaporation rate $\mathrm{J}$ related to the droplet surface area depending on the droplet size, substrate temperature and velocity of the gas flow in minichannel. The curves have a local minimum, whereas it is missing in the evaporation of a droplet in the surrounding atmosphere $[2,3]$. In the initial moment of time the drop takes up to half of the channel height and the gas flow has a significant impact on evaporation. Since the gas velocity in a flat channel has a parabolic profile, the velocity of drop blowing decreases with reduction in the drop height; this explains the local minimum. The same effect is present for the evaporation of droplet blowing by air in the open atmosphere, where a characteristic scale is the boundary layer thickness relative to the droplet height [2, 3]. Following conclusion can be drown that evaporation rate $\mathrm{J}$ is mostly affected by gas velocity for droplets with diameter $\mathrm{d}$ higher than $6 \mathrm{~mm}$ (see in figure 6) and temperature substrate $50^{\circ} \mathrm{C}$ and less, because blue and black symbols have the same values. Further, when the drop becomes flat (see figure 5), that is its diameter several times exceeds the height of the drop, the surface temperature of the drop becomes close to the temperature of the substrate, leading to increased evaporation. We can notice that size of droplet before disappearing depends from temperature; one of the explanations of this fact is that surface tension is reducing with increasing of temperature. 


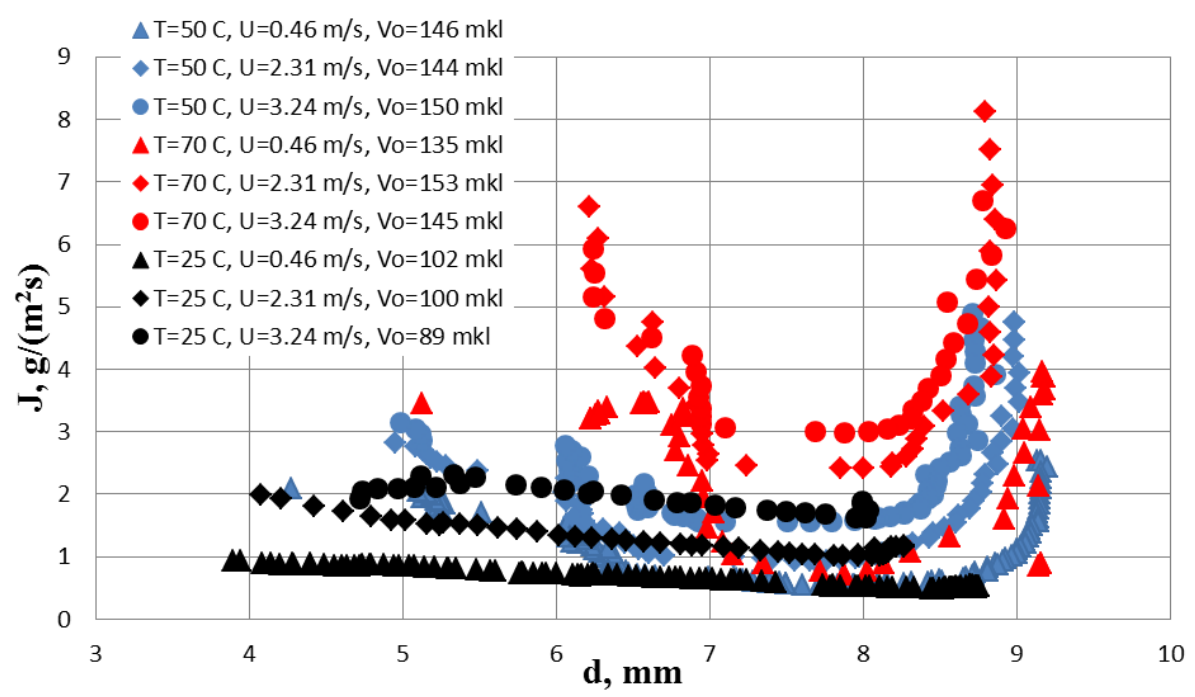

Fig. 6. The evaporation rate versus the droplet diameter. $\mathrm{T}_{\text {gas }}=25^{\circ} \mathrm{C}$.

\section{Conclusion}

In this paper, the evaporation of a single droplet in the $6 \mathrm{~mm}$ channel was studied in detail; for this purpose the images of the drop were recorded with an interval of $10 \mathrm{sec}$ and analyzed. The influence of the substrate temperature, the gas flow rate and temperature on the evaporation of water drops was determined experimentally. We can draw the following conclusions: the evaporation rate is significantly determined by the substrate temperature. It is found local minimum on evaporation rate curves. The gas flow rate also affects the evaporation, and this effect is maximal at the initial moment of time. Air temperature has a little effect to droplet evaporation.

The authors express their gratitude to the Russian Science Foundation for the support of this work (project No. 14-19-01755).

\section{References}

1. J. Kim, Int. J. Heat Fluid Flow 28, 753 (2006)

2. E.Ya. Gatapova, A.A. Semenov, D.V. Zaitsev, O.A. Kabov, Colloids Surf., A 441, 776 (2014)

3. A.A. Semenov, D.V. Feoktistov, D.V. Zaitsev, G.V. Kuznetsov, O.A. Kabov, Thermophys. Aeromech. 22, 771 (2015)

4. O.A. Kabov, D.V. Zaitsev, Doklady Physics 58, 292 (2013)

5. V. Cheverda, I. Marchuc, A.Karachevsky, A. Orlik and O. Kabov, Thermophys. Aeromech. 23, 415 (2016)

6. O.A. Kabov, D.V. Zaitsev, D.P. Kirichenko, V.S. Ajaev, Nanosc. Microsc. Therm. (to be published)

7. Y. Tsoumpas, S. Dehaeck, A. Rednikov, P. Colinet, Langmuir 31, 13334 (2015)

8. L. Bin, R. Bennacer, A. Bouvet, Appl. Therm. Eng. 31, 3792 (2011) 